\title{
Two-band superfluidity and intrinsic Josephson effect in alkaline-earth-metal Fermi gases across an orbital Feshbach resonance
}

\author{
M. Iskin \\ Department of Physics, Koç University, Rumelifeneri Yolu, 34450 Sartyer, Istanbul, Turkey
}

(Received 2 May 2016; published 25 July 2016)

\begin{abstract}
We first show that the many-body Hamiltonian governing the physical properties of an alkaline-earth ${ }^{173} \mathrm{Yb}$ Fermi gas across the recently realized orbital Feshbach resonance is exactly analogous to that of two-band $s$-wave superconductors with contact interactions; i.e., even though the free-particle bands have a tunable energy offset in between and are coupled by a Josephson-type attractive interband pair scattering, the intraband interactions have exactly the same strength. We then introduce two intraband order parameters within the BCS mean-field approximation and investigate the competition between their in-phase and out-of-phase (i.e., the so-called $\pi$ phase) solutions in the entire BCS-BEC evolution at zero temperature.
\end{abstract}

DOI: 10.1103/PhysRevA.94.011604

Introduction. Over the past decade or so, the cold-atom systems have emerged as versatile quantum simulators of fewand many-body physics theories [1-5]. Thanks partly to their high degree of tuning capacities, they proved to be ideal test beds for exploring not only few-body phenomena including the simplest two-body and exotic Efimov bound states $[1,6]$ but also macroscopic phases of matter ranging from BEC and superfluidity of atomic Bose gases, BCS superfluidity and BCS-BEC crossover of atomic Fermi gases, superfluid-Mott insulator transition and quantum magnetism of lattice gases, and topological insulators and superfluids [2-5]. In addition, atomic systems also bridge the gap between the macroscopic properties of many-body systems to microscopic physics of their constituent particles under the same setting, providing hindsights into the realm of mesoscopic systems as well $[7,8]$.

One of the most crucial ingredients behind this success is the ability to control the strength and symmetry of the interparticle interactions with atomic precision [1]. In particular, since alkali atoms (with a single valence electron) have nonzero electronic angular momentum in their ground states, they are highly sensitive to externally applied magnetic fields, and the Zeeman shifts of their different electronic spin states provide a knob to control the inter-hyperfine-state interactions. More specifically, alkali atoms allow for tunable couplings between the energies of a two-body closed-channel bound state and of two interacting open-channel atoms via what is known as the magnetic Feshbach resonance [1]. On the other hand, while the zero electronic angular momentum of ${ }^{173} \mathrm{Yb}$-like alkaline-earth-metal atoms (with two valence electrons) make them highly insensitive to external magnetic fields, it is still possible to control the inter-orbital-state interactions with only tens of Gauss via what is known as the orbital Feshbach resonance [9-13]. Here, it is the Zeeman shifts of different nuclear-spin states of atoms that are used to tune the coupling between closed and open channels. Given that experimentalists are now pursuing the superfluid regime [10-12], the newly realized orbital Feshbach resonance in a ${ }^{173} \mathrm{Yb}$ Fermi gas promises a new wave of interdisciplinary interest for studying many-body phenomena in an uncharted atomic-physics territory due to their direct connection to a wide class of so-called two-band superconductors, which includes many of the recently discovered iron-based superconductors [14-17].
For instance, it turns out that the many-body Hamiltonian governing the physical properties of alkaline-earth-metal Fermi gases across an orbital Feshbach resonance [9,13] is exactly analogous to that of two-band $s$-wave superconductors with contact interactions in one of its most simplest forms [18-21]. That is, even though the free-particle bands are shifted by a tunable energy offset in between and are coupled by an attractive interband pair scattering, the intraband interactions have exactly the same strength. In a broader context, since the two-band Hamiltonian also resembles that of Josephson junctions between two condensates, the physics across an orbital Feshbach resonance can also be thought of as an intrinsic Josephson effect [22-24]. The key role played by the interband Josephson coupling between two intrapaired bands is played in atomic systems by the intraorbital scattering lengths between the open- and closed-channel atoms. Thus, motivated by the recent theoretical and experimental proposals [9-13], here we introduce two intraband order parameters within the BCS mean-field approximation and investigate the competition between their in-phase and out-of-phase solutions in the entire BCS-BEC evolution at zero temperature.

Effective two-band model. To describe alkaline-earth-metal Fermi gases across an orbital Feshbach resonance, we start with the momentum-space Hamiltonian [25]

$$
H=\sum_{i \sigma \mathbf{k}} \xi_{i \mathbf{k}} c_{i \sigma \mathbf{k}}^{\dagger} c_{i \sigma \mathbf{k}}-\sum_{i j \mathbf{q}} V_{i j} b_{i \mathbf{q}}^{\dagger} b_{j \mathbf{q}},
$$

where the band index $i \equiv\{1,2\}$ and pseudospin index $\sigma \equiv$ $\{\uparrow, \downarrow\}$ correspond, respectively, to four-way superpositions of atoms in two different orbital states $\{g, e\}$ with two possible nuclear-spin projections $\{\Uparrow, \Downarrow\}$. As summarized in Fig. 1, the $i=1$ and 2 bands refer, respectively, to the atoms in the open and closed channels. The operator $c_{i \sigma \mathbf{k}}^{\dagger}$ creates a single $\sigma$ particle in band $i$ with momentum $\mathbf{k}$ and dispersion $\xi_{i \mathbf{k}}=$ $\varepsilon_{\mathbf{k}}-\mu_{i}$, where $\varepsilon_{\mathbf{k}}=k^{2} /(2 m)$ is the energy of a free particle (in units of $\hbar=1$ ) and the energy shift (i.e., the detuning parameter $\delta \geqslant 0$ ) is incorporated into the effective chemical potentials as $\mu_{1}=\mu$ and $\mu_{2}=\mu-\delta / 2$.

Since the density-density interactions are typically shortranged in atomic systems, the zero-ranged interactions accounted for by the second term in Eq. (1) may be sufficient for our purposes, where the operator $b_{i \mathbf{q}}^{\dagger}=\sum_{\mathbf{k}} c_{i \uparrow, \mathbf{k}+\mathbf{q} / 2}^{\dagger} c_{i \downarrow,-\mathbf{k}+\mathbf{q} / 2}^{\dagger}$ 


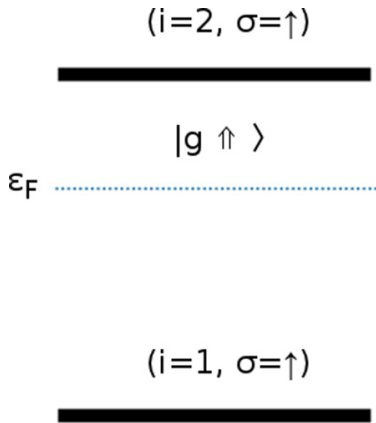

$|e \Uparrow\rangle$ $(i=2, \sigma=\downarrow)$

$|e \Downarrow\rangle$
FIG. 1. Coupling between ${ }^{173} \mathrm{Yb}$ atoms in two orbital states $(g$ and $e$ ) with two nuclear-spin projections ( $\Uparrow$ and $\Downarrow$ ) can effectively be described as a pseudo-spin-1/2 ( $\uparrow$ and $\downarrow$ ) Fermi gas with two nondegenerate bands ( 1 and 2 ). Here, the band offset $\delta / 2$ is a tunable parameter, and the Fermi energy $\varepsilon_{F}$ is the energy scale used in numerics.

creates pairs of $\uparrow$ and $\downarrow$ particles in band $i$ with center-of-mass momentum q. While the intraband interactions turned out to be attractive with equal amplitudes $V_{11}=V_{22}=\left(g_{-}+g_{+}\right) / 2$ $\geqslant 0$, the interband interaction $V_{12}=V_{21}=\left(g_{-}-g_{+}\right) / 2$ may be attractive (positive) or repulsive (negative) depending on the particular species of atom at hand. Here, $g_{ \pm} \geqslant 0$ are the amplitudes for the bare atom-atom attractions in the singlettriplet (antisymmetric-symmetric) superpositions of the orbital degrees of freedom, and they are related to the corresponding scattering lengths $a_{s \pm}$ via the usual renormalization relations $1 / g_{ \pm}=-m \Omega /\left(4 \pi a_{s \pm}\right)+\sum_{\mathbf{k}} m / k^{2}$. Here, $\Omega$ is the volume, and even though both $g_{ \pm}$and $V_{i j}$ depend explicitly on the particular value of cutoff $\left(k_{0}\right)$ used in k-space integrals, our many-body results given below are independent of $k_{0}$ as long as $k_{0} \rightarrow \infty$ is chosen sufficiently high. Therefore, since $V_{12} \gtrless 0$ for $1 / a_{s-} \gtrless 1 / a_{s+}$, we note that $V_{12}$ is positive for a ${ }^{173} \mathrm{Yb}$ Fermi gas where $a_{s+} \approx 1900 a_{0}$ and $a_{s-} \approx 200 a_{0}$ with $a_{0}$ the Bohr radius [10,11].

Two-body bound states in vacuum. It has recently been shown that the two-body $s$-wave scattering length between two open-channel alkaline-earth atoms is given by [9], $a_{s}=$ $a_{s 2}+\sqrt{m \delta} a_{s 1}^{2} /\left(1-a_{s 2} \sqrt{m \delta}\right)$, where $a_{s 2(1)}=\left(a_{s-} \pm a_{s+}\right) / 2$. This shows that $a_{s}$ changes from $a_{s 2}$ to $a_{s+} a_{s-} / a_{s 2}$ as $\delta$ increases from 0 to $\infty$, with an intermediate divergence at $\delta_{\text {res }}=1 /\left(m a_{s 2}^{2}\right)$ when the conditions $a_{s 2}>0$ and $a_{s 1} \neq$ 0 are both satisfied, and an intermediate zero crossing at $\delta_{0}=1 /\left[m\left(a_{s 2}-a_{s 1}^{2} / a_{s 2}\right)^{2}\right]$ when the condition $a_{s 2}>\left|a_{s 1}\right|$ is satisfied. Note that since $a_{s}=a_{s 1}^{2} /\left(a_{s 2} 0^{ \pm}\right)$as $\delta \rightarrow \delta_{\text {res }}^{\mp}$, there are two possible scenarios when $a_{s 2}>0$ and $a_{s 1} \neq 0$. In the first-case scenario, if both $a_{s+}$ and $a_{s-}$ are positive, then increasing $\delta$ first increases $a_{s}$ from $a_{s 2}$ to $+\infty$ and then from $-\infty$ to $a_{s+} a_{s-} / a_{s 2}>0$ with an intermediate zero crossing at $\delta_{0}>\delta_{\text {res. }}$. This is the case for a ${ }^{173} \mathrm{Yb}$ Fermi gas for which $a_{s 2}>\left|a_{s 1}\right|>0$. In the second-case scenario, if only one of $a_{s \pm}$ is negative in such a way that $0<a_{s 2}<\left|a_{s 1}\right|$ then increasing $\delta$ first increases $a_{s}$ from $a_{s 2}$ to $+\infty$ and then from $-\infty$ to $a_{s+} a_{s-} / a_{s 2}<0$ without an intermediate zero crossing.

It is well-known that a two-body bound state in vacuum is characterized by $a_{s}>0$ with binding energy $\varepsilon_{b}=1 /\left(m a_{s}^{2}\right)$.
This suggests that increasing $\delta$ from 0 to $\delta_{\text {res }}^{-}$gradually weakens the binding of atoms as $a_{s}$ increases from $a_{s 2}$ to $+\infty$, beyond which the divergence of $a_{s} \rightarrow-\infty$ as $\delta \rightarrow \delta_{\text {res }}^{+}$signifies the complete unbinding of atoms, i.e., the disappearance of the two-body bound state. In addition, since $a_{s} \rightarrow a_{s 2}$ not only in the $\delta \rightarrow 0$ but also in the $a_{s 1} \rightarrow 0$ limit, the conditions $\delta>0, a_{s 1} \neq 0$, and $a_{s 2}>0$ are all essential requirements for realizing an orbital Feshbach resonance. For instance, it turns out that a ${ }^{173}$ Yb Fermi gas with $a_{s+} \gg a_{s-} \gg a_{0}$ requires such a low $\delta_{\text {res }}$ threshold that it has recently allowed for the very first creation of such a resonance shortly after its theoretical prediction [9-12]. Even though two-body bound states are not allowed in the open channel when $a_{s} \leqslant 0$ (or equivalently $\delta \geqslant$ $\delta_{\text {res }}$ ), many-body bound states may still prevail in the ground state as Cooper pairs, which is discussed next.

Mean-field theory. Motivated by the well-documented success of the simplest BCS-BEC crossover approach developed for one-band Fermi gases across a magnetic Feshbach resonance [3], here we introduce a BCS-like intraband order parameter $\Delta_{i}=-\sum_{j} V_{i j}\left\langle b_{j \mathbf{0}}\right\rangle$ for each band, where $\langle\cdots\rangle$ is a thermal average within the mean-field pairing approximation. Therefore, the complex parameters $\Delta_{1}$ and $\Delta_{2}$ are uniform in space since we set $\mathbf{q}=\mathbf{0}$, and they in general need to be determined together with the corresponding number equations $N_{i}=\sum_{\sigma \mathbf{k}}\left\langle c_{i \sigma \mathbf{k}}^{\dagger} c_{i \sigma \mathbf{k}}\right\rangle$. After some straightforward algebra, the grand potential becomes [26] $\Omega_{m f}=\sum_{i \mathbf{k}}\left[\xi_{i \mathbf{k}}-E_{i \mathbf{k}}-2 T \ln \left(1+e^{-E_{i \mathbf{k}} / T}\right)\right]+\left(V_{22}\left|\Delta_{1}\right|^{2}+\right.$ $\left.V_{11}\left|\Delta_{2}\right|^{2}-V_{12} \Delta_{1}^{*} \Delta_{2}-V_{21} \Delta_{2}^{*} \Delta_{1}\right) /\left(V_{11} V_{22}-V_{12} V_{21}\right)$, and the resultant self-consistency equations can be compactly written as

$$
\begin{gathered}
\Delta_{i}=\sum_{j \mathbf{k}} V_{i j} \frac{\Delta_{j}}{2 E_{j \mathbf{k}}} \tanh \left(\frac{E_{j \mathbf{k}}}{2 T}\right), \\
N_{i}=\sum_{\mathbf{k}}\left[1-\frac{\xi_{i \mathbf{k}}}{E_{i \mathbf{k}}} \tanh \left(\frac{E_{i \mathbf{k}}}{2 T}\right)\right],
\end{gathered}
$$

where $E_{i \mathbf{k}}=\sqrt{\xi_{i \mathbf{k}}^{2}+\left|\Delta_{i}\right|^{2}}$ is the energy of the quasiparticle excitations in the $i$ th band, $T$ is the temperature, and the Boltzmann constant $k_{B}$ is set to unity. The set of coupled equations given above is the generalization of the usual one-band crossover approach to the case of multiband systems, and we hope that this description remain sufficient in understanding (at least qualitatively) some of the low$T$ properties of alkaline-earth-metal Fermi gases across an orbital Feshbach resonance in the entire parameter regime of interest.

In addition to the trivial $\left(\Delta_{1}=\Delta_{2}=0\right)$ one, Eq. (2) allows for two nontrivial solutions with $\Delta_{i}=\left|\Delta_{i}\right| e^{i \varphi_{i}}$, such that the grand potential is minimized or maximized either by the in-phase $\left(\varphi_{1}=\varphi_{2}\right)$ or the out-of-phase $\left(\varphi_{1}=\varphi_{2}+\pi\right)$ one depending on whether the Josephson-type coupling $V_{12}=V_{21}$ is positive or negative as long as the stability condition $V_{11} V_{22}>$ $V_{12} V_{21}$ manifests. As discussed below, the so-called $\pi$-phase solution is directly associated with the recently realized orbital Feshbach resonance in a ${ }^{173}$ Yb Fermi gas $[10,11]$. Thus, the sign of the interband coupling determines the relative phases of the stable (ground state) and unstable (excited state) solutions for a generic two-band model. In contrast to the relative phase, 
the overall phase $\varphi_{1}+\varphi_{2}$ is not a physical observable, and its random value is chosen spontaneously at a given realization. Note that the $V_{12}=V_{21}=0$ limit is not entirely trivial because while $\varphi_{1}$ and $\varphi_{2}$ are completely uncoupled, i.e., they are not physical observables, $\left|\Delta_{1}\right|$ and $\left|\Delta_{2}\right|$ are still coupled when $\delta \neq 0$ even in the $V_{11}=V_{22}$ case considered in this Rapid Communication. In addition, while $\mu$ is varied in such a way to fix the overall number of particles $N=N_{1}+N_{2}$ at a particular value (see the section on numerical results), the relative band population is determined self-consistently for a given $\delta$.

Analytical limits. It is desirable to gain intuitive understanding of the analytically tractable limits as much as possible before going through the cumbersome details of the fully numerical calculations. For instance, we reach the following conclusions by simply analyzing the general structure of Eq. (2).

(1) $g_{ \pm} \rightarrow 0$ or equivalently $1 / a_{s \pm} \rightarrow-\infty$ limit. This leads to a unique in-phase/out-of-phase solution with $\left|\Delta_{1}\right|=$ $\left|\Delta_{2}\right|$ determined by $1=g_{\mp} \sum_{i \mathbf{k}} \tanh \left[E_{i \mathbf{k}} /(2 T)\right] /\left(4 E_{i \mathbf{k}}\right)$.

(2) $g_{+} \rightarrow g_{-}=g$ or equivalently $a_{s+}=a_{s-}$ limit. Since $V_{12}=V_{21}=0$ in this limit, $\varphi_{1}$ and $\varphi_{2}$ are not physical observables, and the nontrivial values for $\left|\Delta_{1}\right|$ and $\left|\Delta_{2}\right|$ are determined by $1=g \sum_{\mathbf{k}} \tanh \left[E_{i \mathbf{k}} /(2 T)\right] /\left(2 E_{i \mathbf{k}}\right)$. This suggests that either $\left|\Delta_{2}\right|$ (ground state) or $\left|\Delta_{1}\right|$ (excited state) vanishes unless $\delta \approx 0$, in such a way that the particles gradually transfer from the upper (lower) to the lower (upper) band with increasing $g$ in the ground (excited) state.

(3) $\delta \rightarrow 0$ limit. Since $V_{11}=V_{22}$ in this Rapid Communication, the degenerate bands become completely symmetric in this limit, giving rise to $\left|\Delta_{1}\right|=\left|\Delta_{2}\right|$ and $E_{1 \mathbf{k}}=E_{2 \mathbf{k}}=$ $E_{\mathbf{k}}$. This leads to a unique in-phase/out-of-phase solution for $g_{\mp}>g_{ \pm}$(or equivalently $1 / a_{s \mp}>1 / a_{s \pm}$ ), where $1=$ $g_{\mp} \sum_{\mathbf{k}} \tanh \left[E_{\mathbf{k}} /(2 T)\right] /\left(2 E_{\mathbf{k}}\right)$.

(4) $\delta \rightarrow \infty$ limit. If $\left|\Delta_{1(2)}\right| \gg\left|\Delta_{2(1)}\right|$ then we obtain $\Delta_{2(1)} / \Delta_{1(2)}=\left(g_{\mp}-g_{ \pm}\right) /\left(g_{-}+g_{+}\right)$. This leads to a unique in-phase (out-of-phase) / out-of-phase (in-phase) solution for $g_{\mp}>g_{ \pm}$. It is clear that these limits are relevant in the neighborhood of $g_{+} \approx g_{-}$(or equivalently $a_{s+} \approx a_{s-}$ ).

(5) $\Delta_{1(2)} \neq 0$ but $\Delta_{2(1)}=0$ limit. This requires $g_{+}=g_{-}=$ $g$ with $1=g \sum_{\mathbf{k}} \tanh \left[E_{1(2) \mathbf{k}} /(2 T)\right] /\left(2 E_{1(2) \mathbf{k}}\right)$, in agreement with the previous limits discussed above.

These analyses clearly show that the allowed nontrivial solutions are unique only in some special limits, and we rely heavily on these analytical limits while characterizing the numerically obtained results in general, as thoroughly discussed next.

Numerical results. In order to obtain cutoff-independent results for $\left|\Delta_{1}\right|,\left|\Delta_{2}\right|$, and $\mu$, we solve the self-consistency Eqs. (2) and (3) with a large k-space cutoff $k_{0}=100 k_{F}$, where the Fermi momentum $k_{F}$ determines the overall density of particles via the usual free-particle relation $n=N / \Omega=$ $k_{F}^{3} /\left(3 \pi^{2}\right)$, written for the lowest band. The corresponding Fermi energy $\varepsilon_{F}=k_{F}^{2} /(2 m)$ is assumed to be less than $\delta / 2$ as illustrated in Fig. 1. Since we iterate $\mu$ to keep $N$ fixed at this value in all of our numerical calculations, here we present only the relative population $\left|N_{1}-N_{2}\right| / N$.

In Fig. 2, we show $\left|\Delta_{1}\right|,\left|\Delta_{2}\right|$, and $\left|N_{1}-N_{2}\right| / N$ for the scattering parameters $a_{s \pm}$ of a ${ }^{173} \mathrm{Yb}$ Fermi gas [10,11]. Depending on the local density of a given experimental setup, one can easily extract the relevant local parameters
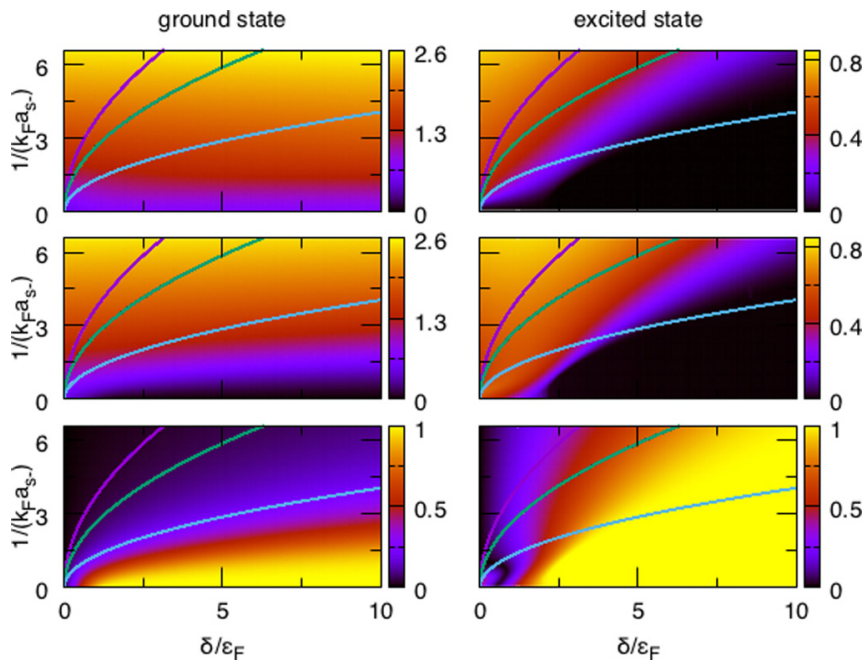

FIG. 2. ${ }^{173} \mathrm{Yb}$ Fermi gas. The colored maps of the magnitudes of the intraband order parameters $\left|\Delta_{1}\right| / \epsilon_{F}$ (top) and $\left|\Delta_{2}\right| / \epsilon_{F}$ (middle) as well as the relative band population $\left|N_{1}-N_{2}\right| / N$ (bottom) are shown for $a_{s+} \approx 1900 a_{0}$ and $a_{s-} \approx 200 a_{0}$. The left and right panels correspond, respectively, to the in-phase and out-of-phase solutions discussed in the text. In addition, $\delta=\delta_{\text {res }}$ (purple), $\delta=2 \delta_{\text {res }}$ (green), and $\delta=\delta_{0}$ (light blue) contours are explicitly shown.

from this figure. For instance, typical atomic systems have densities of order $n \approx 10^{14} \mathrm{~cm}^{-3}$, corresponding to the dimensionless parameters $1 /\left(k_{F} a_{s+}\right) \approx 0.69,1 /\left(k_{F} a_{s-}\right) \approx 6.58$, and $\delta_{\text {res }} / \varepsilon_{F} \approx 3.14$. At resonance, we find $\Delta_{1} \approx 2.55 \epsilon_{F}, \Delta_{2} \approx$ $2.51 \epsilon_{F}, \mu \approx-46 \epsilon_{F}$, and $N_{1}-N_{2}=0.027 N$ for the in-phase (ground-state) solution, and $\Delta_{1} \approx 0.65 \epsilon_{F}, \Delta_{2} \approx-0.76 \epsilon_{F}$, $\mu \approx 0.34 \epsilon_{F}$, and $N_{1}-N_{2}=0.41 N$ for the out-of-phase (excited-state) solution. Away from the resonance, Fig. 2 clearly shows that while the latter solution depends strongly on $\delta$, the former solution does not. For instance, when $\delta=2 \delta_{\text {res }}$, we find $\Delta_{1} \approx 2.57 \epsilon_{F}, \Delta_{2} \approx 2.48 \epsilon_{F}, \mu \approx-46 \epsilon_{F}$, and $N_{1}-N_{2}=0.053 N$ for the in-phase solution, and $\Delta_{1} \approx$ $0.38 \epsilon_{F}, \Delta_{2} \approx-0.53 \epsilon_{F}, \mu \approx 0.79 \epsilon_{F}$, and $N_{1}-N_{2}=0.79 \mathrm{~N}$ for the out-of-phase solution. This suggests that the excitedstate solution is driven mainly by the orbital Feshbach resonance.

To further support this inference, we note that if $a_{s \pm}>$ $a_{s \mp}>0$ then the antisymmetric and symmetric superpositions of the order parameters $\Delta_{ \pm}=\left(\Delta_{2} \mp \Delta_{1}\right) / 2$ are associated, respectively, with the shallow $\left(a_{s \pm}\right)$ and deep $\left(a_{s \mp}\right)$ two-body bound states. In addition, since the shallow one is solely responsible for the orbital Feshbach resonance, these bound states correspond, respectively, to the excited-and ground-state solutions discussed above. In contrast, if only one of $a_{s \pm}$ is positive then the corresponding bound state $a_{s \pm}$, and hence the combination $\Delta_{ \pm}$, is associated with the orbital Feshbach resonance as long as $a_{s 2}>0$. Thus, as a rule of thumb, we conclude that the formation of bound states across an orbital Feshbach resonance is associated with the $\max \left|\Delta_{ \pm}\right|$ superposition whenever $a_{s \pm}>\left|a_{s \mp}\right|$, i.e., the out-of-phase/inphase solution. This analysis again suggests that it is the excited-state (out-of-phase) solution that is predominantly characterized by the orbital Feshbach resonance in a ${ }^{173} \mathrm{Yb}$ Fermi gas. 

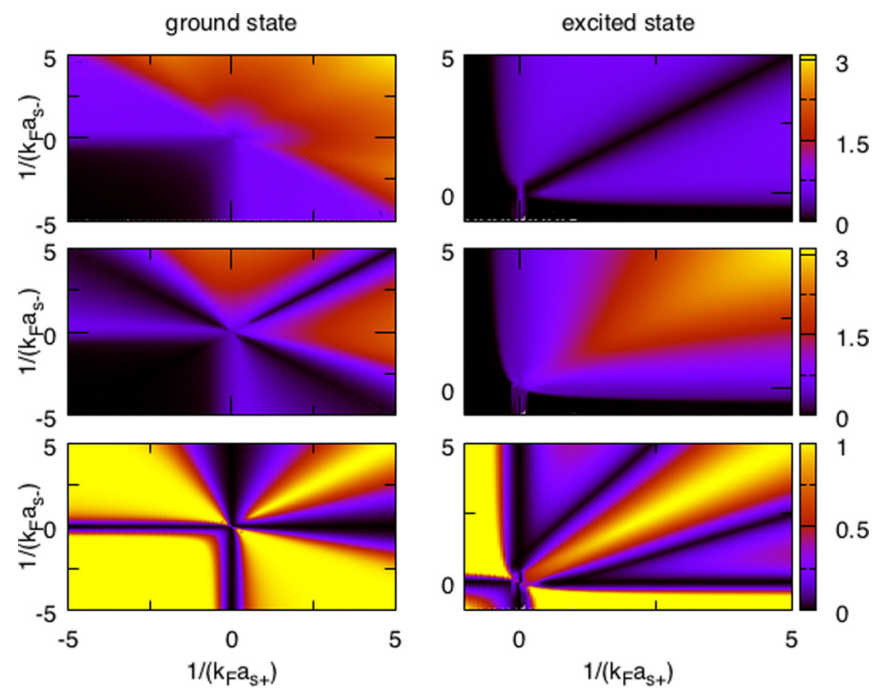

FIG. 3. Resonant Fermi gas. The colored maps of the magnitudes $\left|\Delta_{1}\right| / \epsilon_{F}$ (top), $\left|\Delta_{2}\right| / \epsilon_{F}$ (middle) and $\left|N_{1}-N_{2}\right| / N$ (bottom) are shown for $\delta=1 /\left(m a_{s 2}^{2}\right)$, so that the gas is at resonance in the regions with $a_{s+}+a_{s-}>0$. The left (right) panel corresponds to the in-phase/out-of-phase (out-of-phase/in-phase) solution depending on $1 / a_{s-} \gtrless 1 / a_{s+}$.

In Fig. 3, we show $\left|\Delta_{1}\right|,\left|\Delta_{2}\right|$, and $\left|N_{1}-N_{2}\right| / N$ for a generic Fermi gas with arbitrary $a_{s \pm}$ values when $\delta=$ $1 /\left(m a_{s 2}^{2}\right)$. Since a wide range of parameter regions satisfying the condition $a_{s 2}>0$ correspond to a resonant Fermi gas in general, this figure may serve as a first-hand guide for future experiments with other species of alkaline-earth-metal atoms. In particular, we find that the excited-state (ground-state) solution strengthens (weakens) as $a_{s+} \rightarrow a_{s-}>0$, in agreement with our analytical analyses given above, suggesting that the alkaline-earth-metal Fermi gases with $a_{s+} \approx a_{s-}>0$ are best candidates for investigating many-body phenomena across an orbital Feshbach resonance [27].

Conclusions. To conclude, there is arguably no doubt that the exact analogy between the Hamiltonian of alkalineearth-metal Fermi gases across an orbital Feshbach resonance $[9,13]$ and of two-band $s$-wave superconductors with contact interactions [18] opens yet a new frontier for studying exotic condensed-matter phenomena in atomic systems. In particular, the key role played by the interband pair scattering between two intrapaired bands is played in atomic systems by the intraorbital scattering lengths between the open and closed channels, and the physics is similar in many ways to intrinsic Josephson effect between two condensates [26,28]. This makes atomic settings an ideal test bed for studying the competition between the in-phase and out-of-phase solutions of the superfluid order parameters and their fluctuations. For instance, in addition to the familiar phonon-like in-phase fluctuations of the relative phase, i.e., the massless Goldstone mode, it may be possible to study the long-sought exciton-like out-of-phase fluctuations of the relative phase, i.e., the massive Leggett mode $[19,22,29,30]$.

Acknowledgments. This work is supported by the funding from TÜBITAK Grant No. 1001-114F232 and the BAGEP award of the Turkish Science Academy, and the author thanks Hui Zhai for his correspondence on past and forthcoming developments.
[1] C. Chin, R. Grimm, P. Julienne, and E. Tiesinga, Feshbach resonances in ultracold gases, Rev. Mod. Phys. 82, 1225 (2010).

[2] I. Bloch, J. Dalibard, and W. Zwerger, Many-body physics with ultracold gases, Rev. Mod. Phys. 80, 885 (2008).

[3] S. Giorgini, L. P. Pitaevskii, and S. Stringari, Theory of ultracold atomic Fermi gases, Rev. Mod. Phys. 80, 1215 (2008).

[4] J. Dalibard, F. Gerbier, G. Juzeliunas, and P. Öhberg, Colloquium: Artificial gauge potentials for neutral atoms, Rev. Mod. Phys. 83, 1523 (2011).

[5] V. Galitski and I. B. Spielman, Spin-orbit coupling in quantum gases, Nature (London) 494, 49 (2013).

[6] D. Blume, Few-body physics with ultracold atomic and molecular systems in traps, Rep. Prog. Phys. 75, 046401 (2012).

[7] A. N. Wenz, G. Zürn, S. Murmann, I. Brouzos, T. Lompe, and S. Jochim, From few to many: Observing the formation of a Fermi sea one atom at a time, Science 342, 457 (2013).

[8] G. Zürn, A. N. Wenz, S. Murmann, A. Bergschneider, T. Lompe, and S. Jochim, Pairing in Few-Fermion Systems with Attractive Interactions, Phys. Rev. Lett. 111, 175302 (2013).

[9] R. Zhang, Y. Cheng, H. Zhai, and P. Zhang, Orbital Feshbach Resonance in Alkali-Earth Atoms, Phys. Rev. Lett. 115, 135301 (2015).

[10] G. Pagano, M. Mancini, G. Cappellini, L. Livi, C. Sias, J. Catani, M. Inguscio, and L. Fallani, Strongly Interacting Gas of Two-
Electron Fermions at an Orbital Feshbach Resonance, Phys. Rev. Lett. 115, 265301 (2015).

[11] M. Höfer, L. Riegger, F. Scazza, C. Hofrichter, D. R. Fernandes, M. M. Parish, J. Levinsen, I. Bloch, and S. Fölling, Observation of an Orbital Interaction-Induced Feshbach Resonance in ${ }^{173} \mathrm{Yb}$, Phys. Rev. Lett. 115, 265302 (2015).

[12] See also the viewpoint article by S. Cornish, Viewpoint: Controlling collisions in a two-electron atomic gas, Physics 8 , 125 (2015).

[13] J. Xu, R. Zhang, Y. Cheng, P. Zhang, R. Qi, and H. Zhai, Orbital Feshbach resonance: A wide narrow resonance for higher transition temperature Fermi superfluid, arXiv:1602.06513 (unpublished).

[14] S. Tsuda, T. Yokoya, Y. Takano, H. Kito, A. Matsushita, F. Yin, J. Itoh, H. Harima, and S. Shin, Definitive Experimental Evidence for Two-Band Superconductivity in $\mathrm{MgB}_{2}$, Phys. Rev. Lett. 91, 127001 (2003).

[15] S. Souma, Y. Machida, T. Sato, T. Takahashi, H. Matsui, S.-C. Wang, H. Ding, A. Kaminski, J. C. Campuzano, S. Sasaki, and $\mathrm{K}$. Kadowaki, The origin of multiple superconducting gaps in $\mathrm{MgB}_{2}$, Nature (London) 423, 65 (2003).

[16] F. Hunte, J. Jaroszynski, A. Gurevich, D. C. Larbalestier, R. Jin, A. S. Sefat, M. A. McGuire, B. C. Sales, D. K. Christen, and D. Mandrus, Two-band superconductivity in $\mathrm{LaFeAsO}_{0.89} \mathrm{~F}_{0.11}$ at very high magnetic fields, Nature (London) 453, 903 (2008). 
[17] M. Ruby, B. W. Heinrich, J. I. Pascual, and K. J. Franke, Experimental Demonstration of a Two-Band Superconducting State for Lead using Scanning Tunneling Spectroscopy, Phys. Rev. Lett. 114, 157001 (2015).

[18] H. Suhl, B. T. Matthias, and L. R. Walker, Bardeen-CooperSchrieffer Theory of Superconductivity in the Case of Overlapping Bands, Phys. Rev. Lett. 3, 552 (1959).

[19] M. Iskin and C. A. R. Sá de Melo, BCS-BEC crossover of collective excitations in two-band superfluids, Phys. Rev. B 72, 024512 (2005).

[20] M. Iskin and C. A. R. Sá de Melo, Two-band superfluidity from the BCS to the BEC limit, Phys. Rev. B 74, 144517 (2006).

[21] M. Iskin and C. A. R. Sá de Melo, Evolution of two-band superfluidity from weak to strong coupling, J. Low Temp. Phys. 149, 29 (2007).

[22] A. J. Leggett, Number-phase fluctuations in two-band superconductors, Progr. Theor. Phys. 36, 901 (1966).

[23] R. Kleiner and P. Müller, Intrinsic Josephson effects in high- $T_{c}$ superconductors, Phys. Rev. B 49, 1327 (1994).

[24] P. J. W. Moll, X. Zhu, P. Cheng, H.-H. Wen, and B. Batlogg, Intrinsic Josephson junctions in the iron-based multi-band superconductor $\left(\mathrm{V}_{2} \mathrm{Sr}_{4} \mathrm{O}_{6}\right) \mathrm{Fe}_{2} \mathrm{As}_{2}$, Nat. Phys. 10, 644 (2014).

[25] This representation makes it explicit that the many-body description of an alkaline-earth-metal ${ }^{173}$ Yb Fermi gas across an orbital Feshbach resonance $[9,10,11,13]$ is exactly analogous to that of simplest two-band $s$-wave superconductors with contact interactions [18].
[26] Note that the interband coupling gives rise to a Josephsontype contribution to the grand potential, $-2 V_{12}\left|\Delta_{1} \Delta_{2}\right| \cos \left(\varphi_{1}-\right.$ $\left.\varphi_{2}\right) /\left(V_{11} V_{22}-V_{12}^{2}\right)$, which depends explicitly on the relative phase of the intraband order parameters.

[27] Due to the presence of a confining trap potential, the Fermi gas forms a shell structure where the superfluid core is surrounded by a normal gas that is induced by $\delta \neq 0$ even at $T=0$ (not shown).

[28] The AC Josephson effect. Accordingly, it can be shown that the relative population evolve in time $t$ as $\Delta N=\Delta N_{0}-$ $16 V_{12}\left|\Delta_{10} \Delta_{20}\right| \cos \left(\Delta \varphi_{0}\right) \sin ^{2}(\delta t / 4) /\left[\delta\left(V_{11} V_{22}-V_{12}^{2}\right)\right]$, where $\Delta N_{0}=N_{20}-N_{10}, \Delta \varphi_{0}=\varphi_{20}-\varphi_{10}$, etc., are the equilibrium $(t=0)$ values. Note that this expression is strictly valid for parameter regimes when $\Delta N \approx \Delta N_{0}$ and $\left|\Delta_{i}\right| \approx\left|\Delta_{i 0}\right|$. Thus, for $k_{0} / k_{F} \rightarrow \infty$, the population imbalance $P=\left(N_{2}-N_{1}\right) / N$ evolves, respectively, as $P=$ $P_{0} \mp 3 \pi\left|\Delta_{10} \Delta_{20}\right| /\left(\delta \epsilon_{F}\right)\left[1 /\left(k_{F} a_{s-}\right)-1 /\left(k_{F} a_{s+}\right)\right] \sin ^{2}(\delta t / 4)$ for the in-phase and out-of-phase solutions. Using the parameters given in the text, this reduces to $P=P_{0} \mp$ $55.5\left|\Delta_{10} \Delta_{20}\right| /\left(\delta \epsilon_{F}\right) \sin ^{2}(\delta t / 4)$ for an ${ }^{173}$ Yb Fermi gas.

[29] N. Bittner, D. Einzel, L. Klam, and D. Manske, Leggett Modes and the Anderson-Higgs Mechanism in Superconductors without Inversion Symmetry, Phys. Rev. Lett. 115, 227002 (2015).

[30] Y.-H. Liu, R. M. Konik, T. M. Rice, and F.-C. Zhang, Giant phonon anomaly associated with superconducting fluctuations in the pseudogap phase of cuprates, Nat. Commun. 7, 10378 (2016). 